ARTICLE

Received 5 Dec 2016 | Accepted 25 May 2017 | Published 30 Jun $2017 \quad$ DOl: 10.1038/ncomms16086 OPEN

\title{
Polar rotor scattering as atomic-level origin of low mobility and thermal conductivity of perovskite $\mathrm{CH}_{3} \mathrm{NH}_{3} \mathrm{Pbl}_{3}$
}

Bing Li ${ }^{1}$, Yukinobu Kawakita ${ }^{1}$, Yucheng Liu ${ }^{2}$, Mingchao Wang ${ }^{3}$, Masato Matsuura ${ }^{4}$, Kaoru Shibata ${ }^{1}$, Seiko Ohira-Kawamura', Takeshi Yamada ${ }^{4}$, Shangchao Lin ${ }^{3}$, Kenji Nakajima' \& Shengzhong (Frank) Liu 2,5

Perovskite $\mathrm{CH}_{3} \mathrm{NH}_{3} \mathrm{Pbl}_{3}$ exhibits outstanding photovoltaic performances, but the understanding of the atomic motions remains inadequate even though they take a fundamental role in transport properties. Here, we present a complete atomic dynamic picture consisting of molecular jumping rotational modes and phonons, which is established by carrying out high-resolution time-of-flight quasi-elastic and inelastic neutron scattering measurements in a wide energy window ranging from 0.0036 to $54 \mathrm{meV}$ on a large single crystal sample, respectively. The ultrafast orientational disorder of molecular dipoles, activated at $\sim 165 \mathrm{~K}$, acts as an additional scattering source for optical phonons as well as for charge carriers. It is revealed that acoustic phonons dominate the thermal transport, rather than optical phonons due to sub-picosecond lifetimes. These microscopic insights provide a solid standing point, on which perovskite solar cells can be understood more accurately and their performances are perhaps further optimized.

\footnotetext{
${ }^{1}$ Neutron Science Section, Japan Proton Accelerator Research Complex, Japan Atomic Energy Agency, Tokai, Ibaraki 319-1195, Japan. ${ }^{2}$ Key Laboratory of Applied Surface and Colloid Chemistry, National Ministry of Education, Institute for Advanced Energy Materials, School of Materials Science and Engineering, Shaanxi Normal University, Xi'an 710119, China. ${ }^{3}$ Department of Mechanical Engineering, Materials Science and Engineering Program, FAMU-FSU College of Engineering, Florida State University, Tallahassee, Florida 32310, USA. ${ }^{4}$ Neutron Science and Technology Center, Comprehensive Research Organization for Science and Society, Tokai, Ibaraki 319-1106, Japan. ${ }^{5}$ Dalian Institute of Chemical Physics, Dalian National Laboratory for Clean Energy, Chinese Academy of Sciences, Dalian 116023, China. Correspondence and requests for materials should be addressed to B.L. (email: bing.li@j-parc.jp) or to S.(F.)L.

(email: liusz@snnu.edu.cn).
} 
$\mathrm{O}$ ver last few years, the inorganic-organic hybrid perovskite solar cells have taken the worldwide research community by storm ${ }^{1-3}$.Typically, the energy conversion efficiencies of solar cells based on methylammonium lead iodide $\left(\mathrm{CH}_{3} \mathrm{NH}_{3} \mathrm{PbI}_{3}\right)$ have been improved from the starting $4 \%$ in 2009 to higher than $20 \%$ in 2015 (refs 4,5 ). Very recently, the successes in growth of inch-sized high-quality single crystals and the device integration on wafers have paved the route to large-scale practical photovoltaic applications ${ }^{6,7}$. These hybrid compounds exhibit distinct physical properties from conventional semiconductors. Their hot-phonon bottleneck effect of energetic carriers is obviously stronger than that of GaAs and other inorganic perovskites, which has been attributed to the acoustic-optical phonon up-conversion ${ }^{8-10}$. The mobility of charge carriers is relatively smaller compared with classical semiconductors ${ }^{1-3,11,12}$ and the scattering mechanism is still under debate ${ }^{13-15}$. Resembling the charge transport, the thermal transport is unusual as well. The thermal conductivity is surprisingly low, $\sim 0.5 \mathrm{Wm}^{-1} \mathrm{~K}^{-1}$ for single crystals at room temperature ${ }^{16}$, which is tenfold lower than that of GaAs (ref. 12) and is even lower than that of amorphous silicon ${ }^{17}$.

It is evident that atomic dynamics underlies these peculiarities. However, the atomic-level description of $\mathrm{CH}_{3} \mathrm{NH}_{3} \mathrm{PbI}_{3}$ is complicated by the hybrid nature where both molecular jumping rotations and phonon excitations have to be taken into account. Moreover, these two components also interact via hydrogen bonds between $\mathrm{H}$ and I (refs 18-20). The best approach for this issue is, without a doubt, inelastic neutron scattering (INS). The density functional theory (DFT) lattice dynamics calculations indicate that low-energy phonons are entirely composed of motions of $\mathrm{Pb}$ and I (refs 21-26), which are easily excited at relatively lower temperatures and hence take main parts in determining the physical properties. These phonons can be efficiently probed throughout the Brillouin zones, due to the larger coherent scattering cross-sections of $\mathrm{Pb}$ and $\mathrm{I}$. Simultaneously, the incoherent scattering cross-section of $\mathrm{H}$ assures the individual motions of molecules can be determined by extracting the quasi-elastic broadening underneath the elastic line, which is known as quasi-elastic neutron scattering (QENS) method $^{27}$. In this work, we show the complete phonons and jumping rotational modes across the low-temperature phase transition, which are obtained by measuring a large single crystal at two high-resolution time-of-flight neutron spectrometers that cover a wide energy window ranging from 0.0036 to $54 \mathrm{meV}$. These results are well supported by the complementary molecular dynamics (MD) simulations. It is revealed that the molecular dipole order plays a dominant role in determining charge transport and thermal transport properties of $\mathrm{CH}_{3} \mathrm{NH}_{3} \mathrm{PbI}_{3}$.

\section{Results}

Jumping rotational modes of $\left[\mathrm{CH}_{3} \mathrm{NH}_{3}\right]^{+} \cdot \mathrm{CH}_{3} \mathrm{NH}_{3} \mathrm{PbI}_{3}$ crystallizes in the common perovskite structure where the organic cation $\left[\mathrm{CH}_{3} \mathrm{NH}_{3}\right]^{+}$occupies the centre of the $\mathrm{PbI}_{6}$ octahedral cage, as shown in Fig. 1a. Neutron and X-ray powder diffraction investigations suggest that it undergoes successive phase transitions, from cubic $(P m \overline{3} \mathrm{~m})$ to tetragonal $(\mathrm{I} / \mathrm{mcm})$ at $330 \mathrm{~K}$, and then to orthorhombic (Pnma) at $165 \mathrm{~K}$ (refs 28,29). Our single crystal exhibits a sharp phase transition between 160 and $165 \mathrm{~K}$, as shown in Supplementary Fig. 2, which is in agreement with these reports. While it is believed that this transition is coupled with orientation of $\left[\mathrm{CH}_{3} \mathrm{NH}_{3}\right]^{+}$, current reports on rotational dynamics are contradictory ${ }^{30,31}$, probably owing to the lack of results on single crystals. Given that this compound is extremely sensitive to ambient conditions ${ }^{32,33}$, measurements on a single crystal (with minimized surface contribution) are anticipated to deliver more intrinsic information. Here, the accurate jumping rotational dynamics of molecules is obtained by performing ultrahigh energy resolution QENS measurements on the large single crystal at the backscattering neutron spectrometer, DNA.

First, we demonstrate the jumping rotational dynamics in the orthorhombic phase. As shown in Fig. 2 a, the spectrum at $50 \mathrm{~K}$ is basically described by a delta function convoluted to the instrumental resolution (the full width at half maximum is $\sim 0.0036 \mathrm{meV}$ ). Around $80 \mathrm{~K}$, the QENS signal described by a Lorentzian function is identified underneath the elastic line, indicative of the activation of rotations. The QENS intensity becomes more noticeable at $140 \mathrm{~K}$. The momentum-transfer averaged spectra along [00l] direction are systematically fitted by including a Lorentzian function, a delta function and a constant background, which are convoluted to the instrumental resolution. An example is given at $140 \mathrm{~K}$ in Fig. $2 \mathrm{~b}$. The half width at half maximum of the Lorentzian function, $\Gamma$, is examined as a function of momentum transfer and temperature. As shown in Fig. 2e, $\Gamma$ is almost independent on $l$. Since $\Gamma$ is inversely related to the relaxation time, such a value gives rise to an average relaxation time of 23(1) ps at $140 \mathrm{~K}$. The temperature dependence is fitted to the Arrhenius relation and the activation energy $\sim 47.9(6) \mathrm{meV}$ is obtained (Fig. 2f). The elastic incoherent structure factor (EISF), defined as the ratio between elastic intensity and the sum of QENS and elastic intensity ${ }^{27}$, is shown in Fig. $2 \mathrm{~g}$ at $140 \mathrm{~K}$. It is described by the threefold jumping rotational $\left(C_{3}\right)$ model, in which $\operatorname{EISF}_{C_{3}}=\frac{1}{3}\left[1+2 j_{0}\left(Q d_{\mathrm{H}-\mathrm{H}}\right)\right]^{27}$, where $j_{0}$ is the zeroth-order spherical Bessel function and $d_{\mathrm{H}-\mathrm{H}}$ is the average distance between two adjacent protons, $\sim 1.72 \AA$ in terms of neutron powder diffraction results ${ }^{28}$ (see Supplementary Fig. 3 for details).

In the tetragonal phase, the jumping rotational dynamics becomes more complicated. The spectrum obtained at $180 \mathrm{~K}$ at DNA displays a narrower QENS profile than that at $140 \mathrm{~K}$, as compared in Fig. 2b,c. The relaxation time is determined to be $64(2)$ ps. At the same time, a much broader Lorentzian function is found by using the cold neutron time-of-flight spectrometer AMATERAS, which provides a much wider energy window (Fig. 2d). Such a large width corresponds to a relaxation time as short as $0.71(3)$ ps. Likewise, EISF is calculated based on DNA data by following the same procedure as above ${ }^{27}$. However, the derived EISF is overestimated because QENS intensity of the faster mode is missed in the total intensity. The recent group theory analysis on jumping rotational dynamics of $\mathrm{CH}_{3} \mathrm{NH}_{3} \mathrm{PbI}_{3}$ suggests a coupled $C_{3}$ and fourfold $\left(C_{4}\right)$ mode in the tetragonal phase $^{31}$. Therefore, we compare the derived EISF with this $C_{4} \otimes C_{3}$ model. Shown in Fig. $2 \mathrm{~h}$ is its comparison with the ratio between elastic intensity and total intensity excluded the $C_{3}$ component. The detailed calculation is given in Supplementary Fig. 4. It can be seen that the experimental data is well reproduced in this way, indicating that a $C_{4}$ mode sets in after the phase transition to the tetragonal phase and the $C_{3}$ mode becomes $\sim 30$ times faster. Hereto, we are able to conclude on the jumping rotational dynamics. In the orthorhombic phase, the protons in $\mathrm{CH}_{3}$ and $\mathrm{NH}_{3}$ undergo jumping rotations with respect to the $\mathrm{C}-\mathrm{N}$ axis. The relaxation time is dramatically shortened in the tetragonal phase while the $\mathrm{C}-\mathrm{N}$ axis starts to rotate with respect to the $c$ axis of the crystal structure. The rotational modes are illustrated in Fig. 1b. Our results about the jumping rotational dynamics are in agreement with the recent QENS measurements on a powder sample by Chen et al. ${ }^{31}$, while are different from those by Leguy et al. ${ }^{30}$ in which freezing of modes is not detected. The relaxation time of the $C_{4}$ mode we determined is very close to that identified in previous $\mathrm{MD}$ simulations, $\sim 40$ ps (ref. 34 ) 

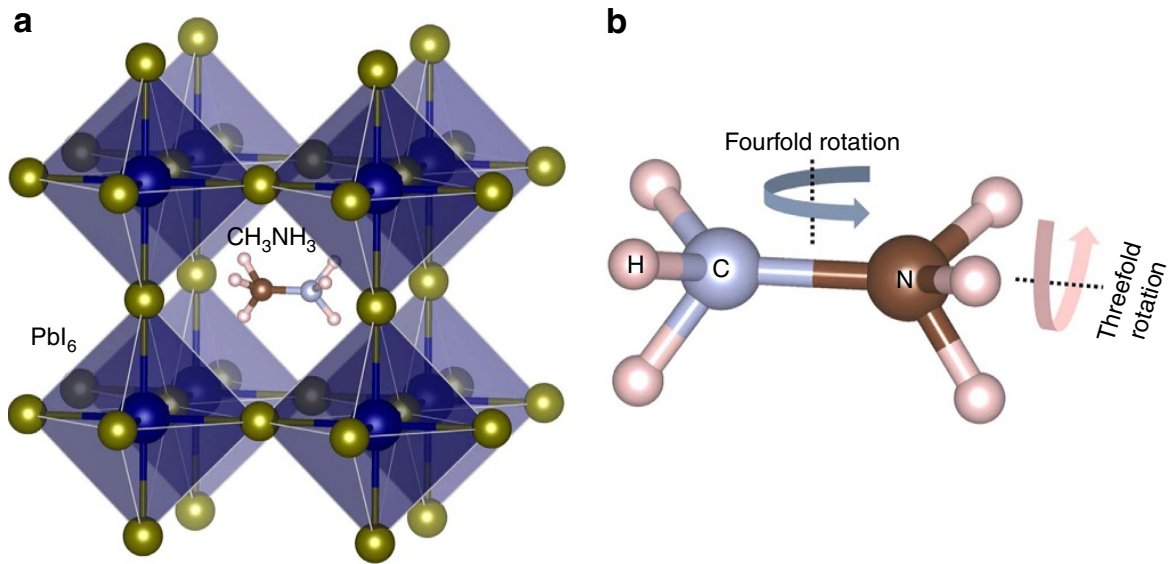

Figure 1 | Crystal structure of $\mathbf{C H}_{\mathbf{3}} \mathbf{N H}_{\mathbf{3}} \mathbf{P b} \mathbf{3}_{\mathbf{3}}$ (a) The schematic diagram of the perovskite structural unit. The organic cation is located in the centre of $\mathrm{Pbl} 6$ octahedral cage. The real structures are complex due to the orientational disorder of organic cations. (b) Geometry of the molecule with schematic drawing of the threefold and fourfold jumping rotational modes.

a

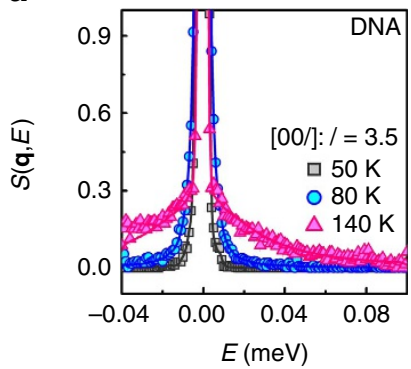

e

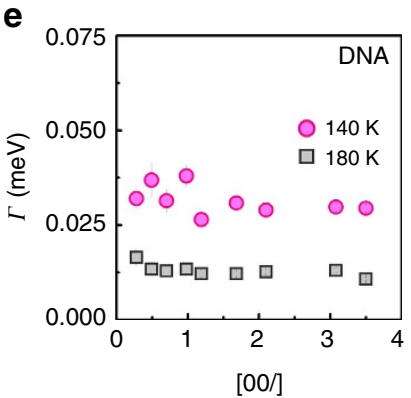

b

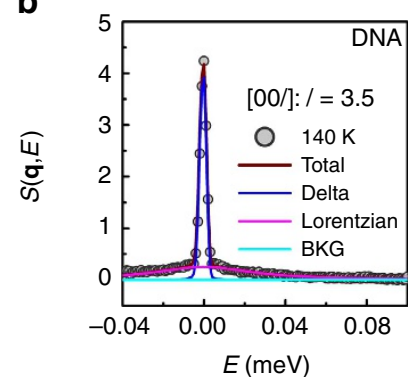

$\mathbf{f}$

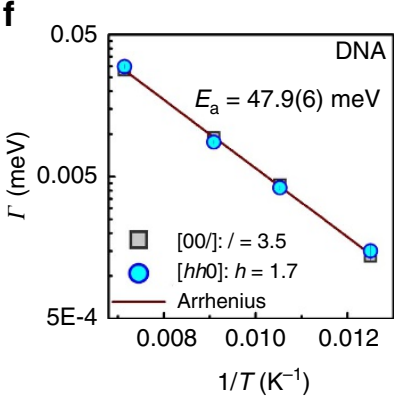

c

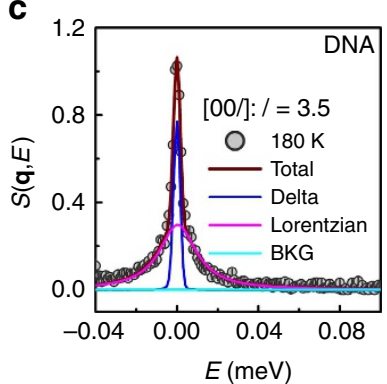

g

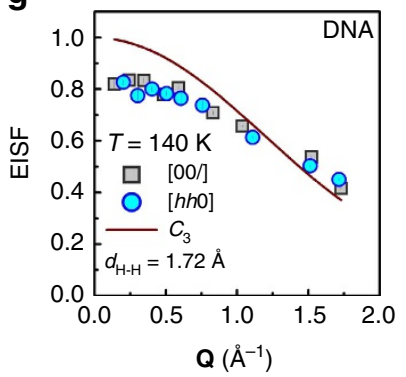

d

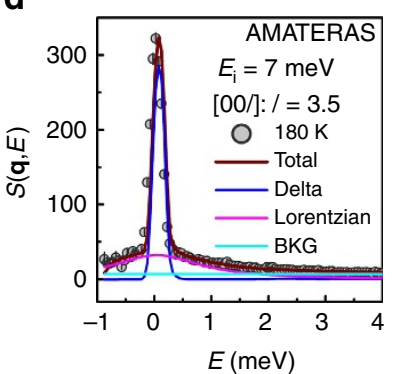

$\mathbf{h}$

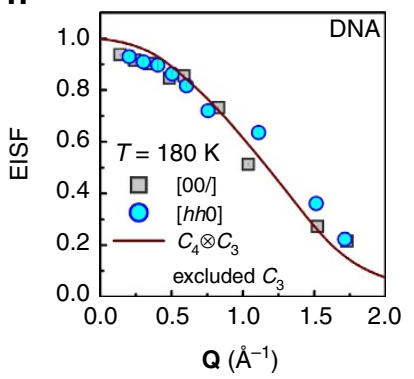

Figure 2 | Jumping rotational dynamics of molecules in $\mathbf{C H}_{\mathbf{3}} \mathbf{N H}_{\mathbf{3}} \mathbf{P b} \mathbf{3}_{\mathbf{3}}$ (a) $\mathbf{S}(\mathbf{q}, E)$ at $I=3.5$ along [00/] at 50, 80 and $140 \mathrm{~K}$ obtained at DNA. (b) The spectral fitting of $S(\mathbf{q}, E)$ at $140 \mathrm{~K}$ for $I=3.5$ along [00/] direction, by including a delta function, a Lorentzian function and a constant background (BKG). $(\mathbf{c}, \mathbf{d})$ The spectral fitting of $S(\mathbf{q}, E)$ obtained at DNA and at AMATERAS at $I=3.5$ along [00/] direction at $180 \mathrm{~K}$, respectively. (e) The half width at half maximum $(\Gamma)$ of the Lorentzian components derived in the fitting, as a function of momentum transfer at 140 and $180 \mathrm{~K}$. (f) The temperature dependence of $\Gamma$ for the orthorhombic phase, fitted to Arrhenius relation $\Gamma \propto \exp \left(-\frac{E_{a}}{k_{B} T}\right)$, where $E_{\mathrm{a}}$ is the activation energy of the jumping rotational mode and $k_{\mathrm{B}}$ is Boltzmann constant. ( $\mathbf{g}, \mathbf{h}$ ) the EISF at 140 and $180 \mathrm{~K}$ compared with $C_{3}$ model and $C_{4} \otimes C_{3}$ model, respectively. In the latter, the $C_{3}$ component is excluded. To compare two directions, the real momentum transfer $\mathbf{Q}$ is used.

Phonons of the orthorhombic and tetragonal phases. The jumping rotational dynamics of an individual molecule is well understood above and now we move to the collective dynamics, that is, phonons, which are measured by selecting several incident energy of neutrons $\left(E_{\mathrm{i}}\right)$ at AMATERAS. The obtained dynamic structure factor $S(\mathbf{q}, E)$ is shown in Fig. 3 at $5 \mathrm{~K}$ in the direction of $[00 l]$. With $E_{\mathrm{i}}$ of $54 \mathrm{meV}$, the full view of $S(\mathbf{q}, E)$ is provided. The momentum-transfer averaged spectrum at $10<l<14$ (Fig. 3d) indicates six prominent modes located at 11.3, 15.3, 18.3, 22.6, 26.0 and $37.6 \mathrm{meV}$, respectively. Except them, there are several less obvious shoulders or humps, as arrowed in the inset. With smaller $E_{\mathrm{i}}$, more details are revealed. We find the peak at
$11.3 \mathrm{meV}$ shown in Fig. 3d is actually a superposition of three peaks at 10.6, 11.5 and $12.6 \mathrm{meV}$ (Fig. 3b,e). There seems a gap located between 6 and $10 \mathrm{meV}$, but several weaker modes are roughly identified as highlighted in the inset. The data with $E_{\mathrm{i}}$ of $7 \mathrm{meV}$ (Fig. 3c,f) suggests four low-energy modes at 2.28, 3.11, 3.79 and $4.35 \mathrm{meV}$. Thanks to the high-resolution, we are able to identify several modes that are overlooked in the previous Raman scattering ${ }^{26}$ and INS measurements ${ }^{21}$. Particularly, the latter and the associated DFT calculations are compared with our experimental results and MD simulations, as listed in Table 1. The numbers correspond to ones appearing in the square brackets of the labels in Fig. $3 \mathrm{~d}-\mathrm{f}$ and Supplementary Fig. 6. It 

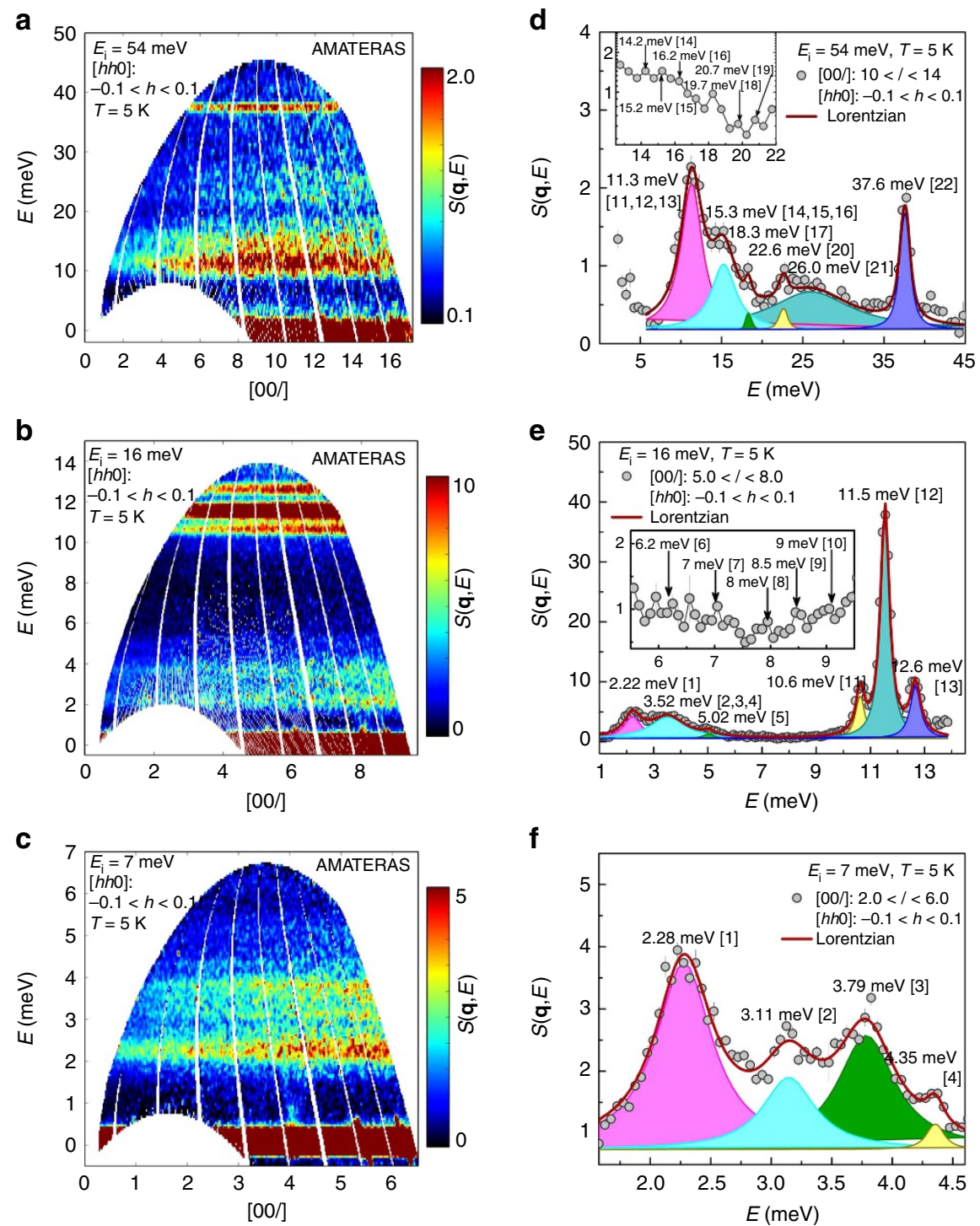

Figure 3 | Longitudinal phonon spectra at $\mathbf{5} \mathbf{K}$ along [00/] obtained at AMATERAS. (a-c) The contour plots of $S(\mathbf{q}, E)$ obtained with $E_{\mathrm{i}}$ of 54,16 and $7 \mathrm{meV}$. (d-f) Momentum-transfer averaged spectra and multiple Lorentzian-function fitting to highlight the energies of modes. The peak position for each component is labelled. The insets highlight small shoulders or humps overlooked by the fitting. For the case of [hhO], please refer to Supplementary Fig. 5 .

can be seen that our measurements match well with the simulations, except the modes 21 and 22, which are dominated by the molecular twist motion ${ }^{24}$. This discrepancy is also reported in another $\mathrm{MD}$ simulation study using the same potential functions ${ }^{24}$, and might be related to the zero-point-energy fluctuations that lead to more lattice distortions as suggested in the DFT calculations on $P 1$ symmetry ${ }^{21}$.

Detailed acoustic phonons of the Brillouin zone (220) are examined with $E_{\mathrm{i}}$ of $4 \mathrm{meV}$. Shown in Fig. $4 \mathrm{a}-\mathrm{d}$ are the longitudinal and transverse phonons at 5 and $180 \mathrm{~K}$, respectively. Even though the background level is high, the dispersions can be seen in both directions. At $5 \mathrm{~K}$, the dispersion curve of longitudinal acoustic (LA) phonon emanates from zone centre of (220), undergoes a steep increase and finally approaches the top in the middle of zone centre and zone boundary ( $h$ about 0.2 ), where it is overlapped with longitudinal optical (LO) phonons. The dispersion of transverse acoustic (TA) phonon exhibits a moderate slope. Intense transverse optical (TO) phonons are also present. At $180 \mathrm{~K}$, the intensity of phonons is fairly enhanced and we determine the accurate dispersions of LA and TA phonons by fitting the momentum-transfer (filled circle) or energy-transfer (filled square) averaged spectra. The solid lines represent the slopes of dispersion curves near the zone centres, which determine the group velocities: $v_{\mathrm{LA}}=2841 \mathrm{~ms}^{-1}$ and $v_{\mathrm{TA}}=1155 \mathrm{~m} \mathrm{~s}^{-1}$. Shown in Fig. $4 \mathrm{e}$ is the fitting of the TA phonon, which yields the lifetime of 3.61(42) ps in the middle of the zone boundary and zone centre. For the LA case, we determine that the lifetime is $\sim 4.39(46)$ ps at $180 \mathrm{~K}$ in the vicinity of the zone centre (Supplementary Fig. 7). Recently, Whalley et al. ${ }^{35}$ have calculated the lifetimes by considering the phonon-phonon interaction. At $300 \mathrm{~K}$, the lifetimes of phonons range around $2 \mathrm{ps}$, which is very close to our results. The product of lifetime and group velocity defines mean-freepath. At $180 \mathrm{~K}$, short lifetimes and small velocities yield mean-free-paths of $\sim 125$ and $42 \AA$ for the LA phonon and TA phonon, respectively. Since we have first determined these critical quantities, it is allowed to compare with previous theoretical counterparts. Listed in Table 2 is the comparison of velocities and mean-free-paths of acoustic phonons ${ }^{35-38}$. It is worth noting that our INS results are well supported by the MD simulations based on the same potential functions as we used here $\mathrm{r}^{34,38}$ 


\begin{tabular}{|c|c|c|c|c|}
\hline No. & INS (powder) ${ }^{21}$ & INS (crystal; this work) & DFT $(\boldsymbol{P} \mathbf{1})^{21}$ & MD (this work) \\
\hline 2 & - & 25.1 & - & 27 \\
\hline 3 & 30 & 30.6 & 28 & 31 \\
\hline 4 & - & 35.1 & - & 35 \\
\hline 5 & 40 & 40.5 & 38 & 39 \\
\hline 8 & 66 & 65 & 58 & 63 \\
\hline 9 & - & 69 & - & 69 \\
\hline 10 & - & 73 & - & 73 \\
\hline 11 & 87 & 85.5 & 68 & 83 \\
\hline 12 & 93 & 92.8 & 79 & 95 \\
\hline 13 & 102 & 101.6 & 90 & 102 \\
\hline 18 & - & 159 & - & 153 \\
\hline 19 & 173 & 167 & 153 & 173 \\
\hline 20 & 182 & 182.3 & 157 & 180 \\
\hline 21 & - & 209.7 & - & 250 \\
\hline 22 & 304 & 303.3 & 302 & $341,352,368$ \\
\hline
\end{tabular}

The INS results at $5 \mathrm{~K}$ are taken from Fig. 3 while the MD results at $100 \mathrm{~K}$ from Supplementary Fig. 6 . The numbers correspond to ones appearing the square brackets in these two figures. The data on a powder sample is from ref. 21 along with the associated with DFT calculations on P1 model.

More strikingly, the LO and TO phonons around $2.28 \mathrm{meV}$ completely vanish at $180 \mathrm{~K}$. The data of the (004) zone (Supplementary Fig. 8) indicates that they survive at $150 \mathrm{~K}$. Thus, we compare the optical phonons located at 10.7 and $12.6 \mathrm{meV}$ at 5, 150 and $180 \mathrm{~K}$, which are two of strongest optical phonons observed in the spectra. At $5 \mathrm{~K}$, these optical phonons are well-defined, as shown in Fig. 3e. With warming up to $150 \mathrm{~K}$, the lifetimes of TO phonons (Fig. 4f) at 10.7 and $12.6 \mathrm{meV}$ are reduced down to $0.91(15)$ and $0.62(7) \mathrm{ps}$ while those of LO phonons (Fig. $4 \mathrm{~g}$ ) are reduced down to $0.82(5)$ and $0.57(2) \mathrm{ps}$, respectively. These lifetimes are much shorter than $5.40(88)$ ps for the TA phonon (inset of Fig. 4e). At $180 \mathrm{~K}$, just above the phase transition, both TO and LO phonons are too broadened to be distinguished. This means the actual lifetimes at $180 \mathrm{~K}$ are much shorter than those at $150 \mathrm{~K}$. This dramatic broadening is greatly consistent with the Raman scattering study in which the lifetime is shortened from 0.6 to 0.15 ps (ref. 26). The characteristic timescales of jumping rotational modes and phonons are summarized in Table 3.

MD simulations of phonons through the phase transition. The finite-temperature phonon excitations are considered in classical MD simulations using recently developed MYP potential functions ${ }^{34}$. In Fig. 5a, we show the phonon density of state (DOS) at $100 \mathrm{~K}$. It is clear that motions of the inorganic part are dominant in the low-energy region while the high-energy spectrum is mostly contributed by molecular motions. The energy of each mode is determined in Supplementary Fig. 6 and compared with the experimental counterpart in Table 1 . The distinct changes of lifetimes of acoustic and optical phonons through the phase transition are consistent with the simulations where the DOS spectrum of optical phonons is much more broadened than that of acoustic ones. As shown in Fig. 5b, the peaks of optical phonons (between 2 and $16 \mathrm{meV}$ ) are remarkably broadened in the tetragonal phase, in agreement with the broadening shown in Fig. 4f,g (for medium-energy optical phonons) and Supplementary Fig. 8 (for low-energy optical phonons). Instead, acoustic phonons shown in the inset are still distinguishable even if their intensities are much weaker than those of optical phonons. The significantly reduced lifetimes of optical phonons in the tetragonal phase imply an additional decay channel taking effect except the phonon-phonon interaction. Whalley et al. ${ }^{35}$ have considered the phonon-phonon interaction and directly calculate the lifetimes. It is shown that there are similar lifetimes for phonons with frequencies below $\sim 3.5 \mathrm{THz}(\sim 14 \mathrm{meV})$. Namely, the phonon decay based on anharmonicity in this energy region is not selective. In contrast, the MD simulations including the long-range electrostatic interactions indicate that optical phonons are more broadened than acoustic ones. Note that the experimentally observed dipole order has been reproduced using the same methodology of simulations ${ }^{34}$. Thus, the additional scattering is most likely attributed to the activated orientational disorder of dipoles in the tetragonal phase, which is similar to the phonon scattering by paraelectric centres ${ }^{39,40}$.

\section{Discussion}

In the tetragonal phase, the temperature dependence of mobility shows a $-3 / 2$ power-law, which was initially attributed to the scattering by acoustic phonons ${ }^{13}$. Later, both the experimental investigation of photoluminescence spectra and the transport theoretical study manifest the Fröhlich interaction between charge carriers and LO phonons is the major scattering mechanism ${ }^{14,15}$. Indeed, considering the deformation potential and the piezoelectric potential as major mechanisms, the calculated mobility is much larger than experimentally measured one $\mathrm{Al}^{4,42}$, indicating that acoustic phonons are less important. Notwithstanding, it is still unclear why the mobility of hybrid perovskites is quite small compared with other inorganic semiconductors that have comparable Fröhlich coupling strengths ${ }^{14}$. More importantly, such a scenario is not able to reproduce the critical-like temperature dependence of mobility at the orthorhombic-to-tetragonal phase transition ${ }^{43}$.

According to the current structural model, the orthorhombic phase is antiferroelectric ${ }^{28}$. Our QENS data suggests that 

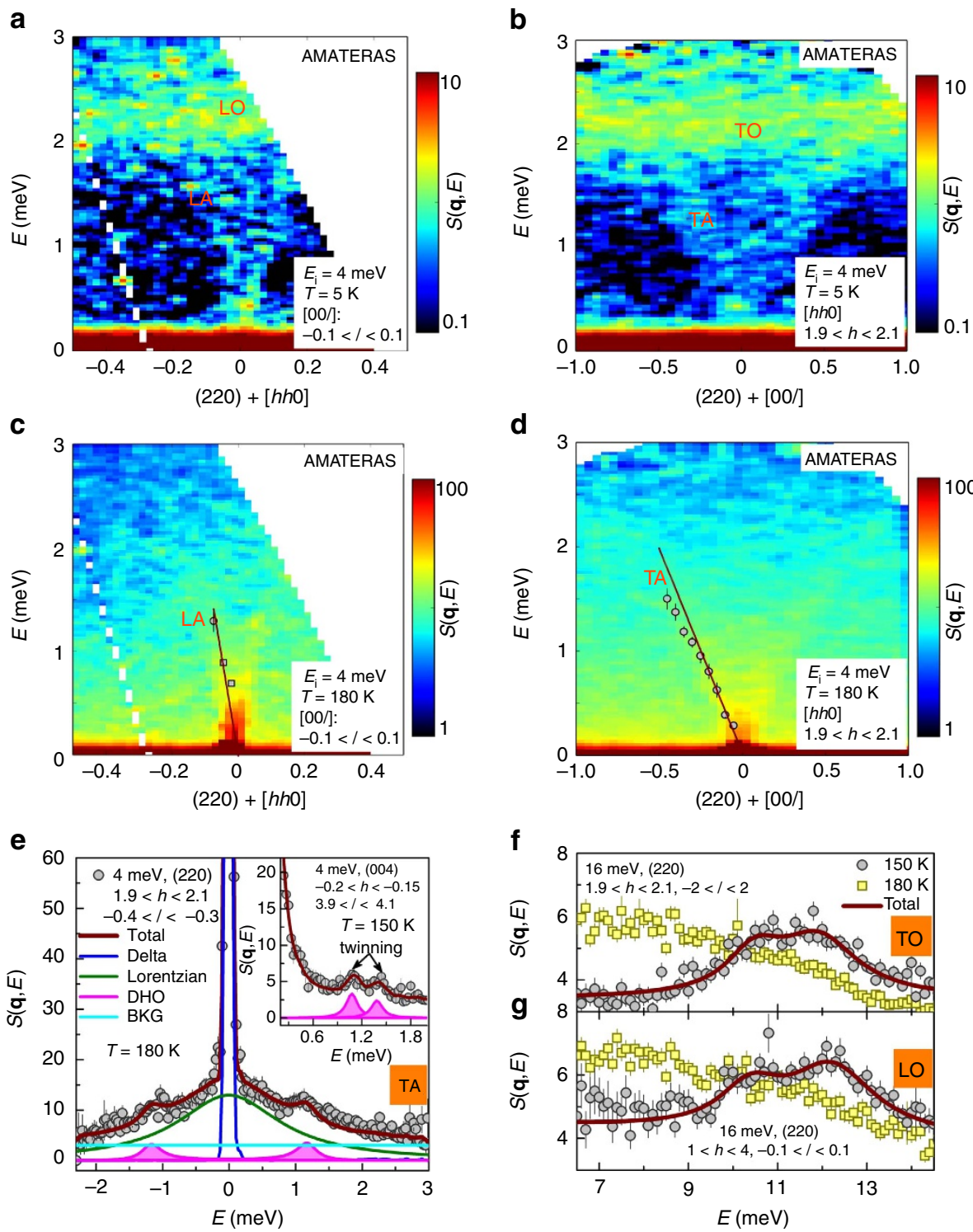

Figure 4 | Phonon broadening at the orthorhombic-to-tetragonal phase transition. (a,c) Longitudinal phonons at 5 and $180 \mathrm{~K}$ of the (220) Brillouin zone; $(\mathbf{b}, \mathbf{d})$ Transverse phonons at 5 and $180 \mathrm{~K}$ of the (220) Brillouin zone. TA, TO, LA and LO phonons are labelled. The filled circles and square in $\mathbf{c}$ and $\mathbf{d}$ are dispersions determined by fitting energy-transfer averaged and momentum-transfer averaged spectra, respectively. The solid lines represent the slopes as approaching the zone centre, which characterize the group velocities. (e) Fitting of momentum-transfer averaged TA phonon spectrum of the (220) Brillouin zone at $180 \mathrm{~K}$. The Lorentzian and damped harmonic oscillator (DHO) functions describe the quasi-elastic component and the TA phonon, respectively. The inset shows the TA phonon at $150 \mathrm{~K}$ of the (004) Brillouin zone. The presence of two peaks is because the crystal is twinned in the orthorhombic phase (also see Supplementary Fig. 11). (f) Fitting of momentum-transfer averaged TO and ( $\mathbf{g}$ ) LO phonons spectra of the (220) Brillouin zone at 150 and $180 \mathrm{~K}$ with $E_{\mathrm{i}}$ of $16 \mathrm{meV}$.

Table 2 | Comparison of properties of acoustic phonons for the tetragonal phase.

\begin{tabular}{|c|c|c|c|c|}
\hline \multicolumn{2}{|c|}{ Velocity $\left(\mathrm{m} \mathrm{s}^{-1}\right)$} & \multicolumn{2}{|c|}{ Mean-free-path $(\AA)$} & Methods \\
\hline 970 & 3,270 & - & - & DFT $(\text { ref. } 36)^{\star}$ \\
\hline$\sim 1,500$ & $\sim 2,800$ & \multirow{2}{*}{\multicolumn{2}{|c|}{$\begin{array}{c}<100 \\
-\end{array}$}} & MD (ref. 38) \\
\hline \multicolumn{2}{|c|}{$\sim 1,200$} & & & Transport (ref. 37) \\
\hline 1,155 & 2,841 & 42 & 125 & INS (this work) \\
\hline
\end{tabular}


Table 3 | Timescales of atomic dynamics and related physical properties.

\begin{tabular}{|c|c|c|c|c|c|c|}
\hline \multirow[t]{2}{*}{ Phases } & \multicolumn{2}{|c|}{ Relaxation time of rotational modes (ps) } & \multicolumn{2}{|c|}{ Lifetime of phonons (ps) } & \multirow[t]{2}{*}{$\kappa\left(\mathbf{W} \mathbf{m}^{-1} \mathbf{K}^{-1}\right)^{16}$} & \multirow[t]{2}{*}{$\mu\left(\mathbf{c m}^{2} v^{-1} s^{-1}\right)^{43}$} \\
\hline & $C_{3}$ & $C_{4}$ & TA & TO (12.6 meV) & & \\
\hline Orthorhombic $(150 \mathrm{~K})^{\star}$ & $23(1)$ & Frozen & $5.40(88)$ & $0.62(7)$ & $\sim 0.75$ & $\sim 4.5$ \\
\hline Tetragonal $(180 \mathrm{~K})$ & $0.71(3)$ & $64(2)$ & $3.61(42)$ & $<<0.62$ & $\sim 0.65$ & $\sim 2.2$ \\
\hline
\end{tabular}

The orientational disorder of dipoles sets in at the transition, where lifetimes of optical phonons and mobility $(\mu)$ undergo giant changes while acoustic phonons and thermal conductivity $(\kappa)$ are less susceptible.

*Relaxation times of rotational modes for orthorhombic phase are taken at $140 \mathrm{~K}$.
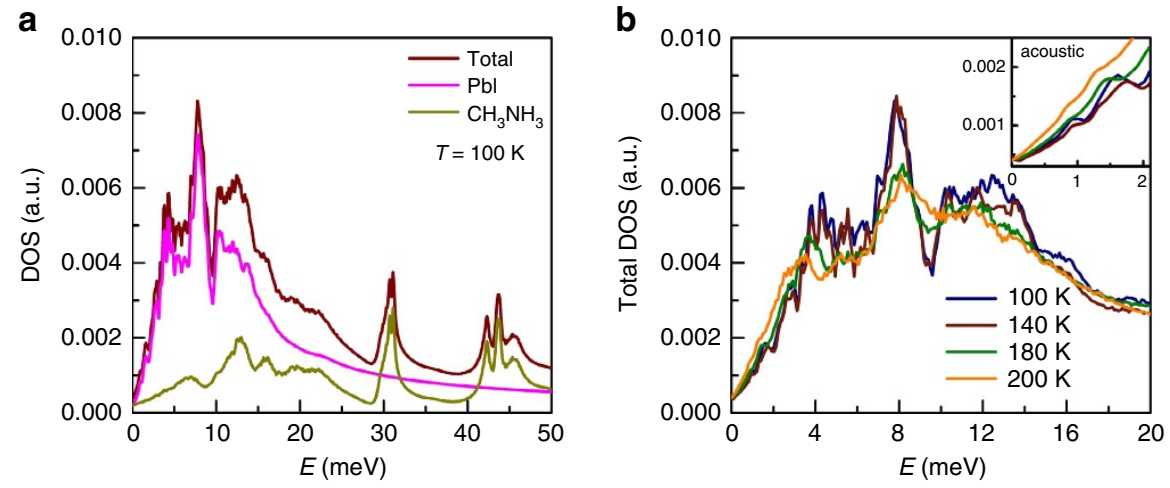

Figure 5 | Phonon DOS calculated in simulations. (a) The total and partial (contributed by the inorganic and molecular species) DOS at $100 \mathrm{~K}$. (b) The total DOS at 100, 140, 180 and $200 \mathrm{~K}$. The inset shows the acoustic phonons located in the low-energy region, which are less broadened than the optical ones during the orthorhombic-to-tetragonal phase transition.

the dipoles are disordered in the picosecond temporal scale in the tetragonal phase. Thus, this orthorhombic-to-tetragonal phase transition is indeed an order-to-disorder transition of dipoles, just as the dielectric constant displays a sharp peak at the transition temperature ${ }^{44}$. Namely, $\mathrm{CH}_{3} \mathrm{NH}_{3} \mathrm{PbI}_{3}$ is not only a polar semiconductor like GaAs, but it is also antiferroelectric. Extensive earlier reports on electrical transport properties of ferroelectric perovskite oxides and IV-VI semiconductors indicate the critical-point polarization fluctuations result in a singularity of resistivity at the transition temperatures, that is, a positive resistivity-temperature slope as approaching the transition temperature from the low-temperature side ${ }^{45-47}$. Similarly, the dipole scattering is also active in hybrid perovskites with polar cations. The important role of molecular dipoles is manifested at the sharp difference of the magnitude of mobility of hybrid $\mathrm{CH}_{3} \mathrm{NH}_{3} \mathrm{PbBr}_{3}$ and all-inorganic $\mathrm{CsPbBr}_{3}$ (ref. 43). At $\sim 120 \mathrm{~K}$ where both compounds crystallize in orthorhombic structures, the mobility of the former is about 5 times lower. In addition, the critical anomaly has been analytically solved for antiferroelectrics, given as $\frac{\mathrm{d} \rho_{\text {crit }}}{\mathrm{d} T} \propto\left(\frac{T}{T_{\mathrm{c}}}-1\right)^{-\alpha}$ at $T>T_{\mathrm{c}}$ and as $\frac{\mathrm{d} \rho_{\text {cit }}}{\mathrm{d} T} \propto\left(1-\frac{T}{T_{\mathrm{c}}}\right)^{2 \beta-1}$ at $T<T_{\mathcal{c}}$, where $\rho_{\text {crit }}$ is critical resistivity, $T_{\mathcal{c}}$ is transition temperature, $\alpha$ and $\beta$ are critical exponents ${ }^{47}$. Thus, one obtains $\mu \propto-\left(1-\frac{T}{T_{c}}\right)^{-2 \beta}$ at $T<T_{\mathrm{c}}$. We find the temperature dependence of mobility near the phase transition of $\mathrm{CH}_{3} \mathrm{NH}_{3} \mathrm{PbI}_{3}$ can be well reproduced with an extremely small value of $\beta$ (Supplementary Fig. 9), indicative of the first-order nature ${ }^{48}$. The sharp change of mobility at the phase transition well coincides with the activation of dipole disorder (see Table 3).

Even though the molecular dipoles are disordered on atomic level as they undergo ultrafast $C_{4}$ jumping rotational mode in the picosecond temporal scale, it is possible that there are nanoscale/mesoscale correlations with slower dynamics ${ }^{49}$. This kind of large-scale structures are compatible with ferroelectric domains predicted in $\mathrm{DFT}^{50}$ and observed using piezoelectric force microscopy ${ }^{51}$. They are perhaps relevant to the polaron picture proposed for hybrid perovskites ${ }^{52}$, which is the key to understand the extremely low recombination rate of carriers.

It is known that lattice thermal conductivity is equal to $1 / 3 C v^{2} \tau$ where $C$ is the lattice specific heat, $\tau$ is the average group velocity and $\tau$ is the average lifetime of phonons ${ }^{53}$. Even through the origin of ultralow thermal conductivity is naturally ascribed to either lower bulk modulus (proportional to $v^{2}$ ) or shorter $\tau$, the profound mechanism is still unclear due to the lack of the complete phonon data, especially acoustic phonons. Our INS study suggests the contribution of optical phonons to thermal transport is marginal because of the significantly shortened lifetimes. On the contrary, the thermal transport is dominated by acoustic phonons. Their nanoscale mean-free-paths together with smaller velocities are responsible for such a small thermal conductivity. Experimentally, the thermal conductivity exhibits a smooth temperature dependence except an abrupt discontinuity at the transition owing to the anomaly of the specific heat ${ }^{16}$ (Table 3 ). This is well consistent with the less varied lifetimes of acoustic phonons across the phase transition. If the optical phonons were major heat carriers, a dramatic drop of thermal conductivity would be expected at the transition. Molecular vibrations have been recognized to be detrimental to thermal transport $35,54,55$. The anharmonic DFT calculations by Whalley et al. ${ }^{35}$ and MD simulations with MYP potential functions by Caddeo et al. ${ }^{54}$ suggest the scattering of low-energy phonons (mostly dominated by the inorganic part) by molecular vibrations acts as the major decay channel, but in another simulation by Hata et al..$^{55}$ molecular vibrations only mediate the interaction between phonons consisting of $\mathrm{PbI}$ motions. Our results seem to agree with the first case because the twist mode at $37.6 \mathrm{meV}$ is significantly broadened even in the orthorhombic phase (Supplementary Fig. 10).

Given that the molecular vibrations (high-energy optical phonons) are very flat modes, their interaction with acoustic phonons is in analogy to the rattling-mode-phonon coupling in the paradigm of electron-crystal phonon-glass ${ }^{56}$, where less dispersive modes 
highly scatter acoustic phonons, leading to glassy-like thermal conductivity $^{57}$. It is considerably promising to achieve superior thermoelectric materials in hybrid inorganic-organic perovskites by enhancing charge transport. Simulations indicate the thermoelectric figure-of-merit of $\mathrm{CH}_{3} \mathrm{NH}_{3} \mathrm{PbI}_{3}$ can reach to 3 at $600 \mathrm{~K}$ with optimal carrier density of $10^{19} \mathrm{~cm}^{-3}$ (ref. 58). It is also predicted that $\mathrm{CH}_{3} \mathrm{NH}_{3} \mathrm{SnI}_{3}$ may have much higher value of figure-of-merit than $\mathrm{Pb}$ case $^{59}$. Moreover, the low thermal conductivity does not only directly enable hybrid perovskites to be appealing thermoelectric materials, but also plays a fundamentally important part in photovoltaic properties. The suppressed thermal conduction promotes the acoustic-optical phonon up-conversion, benefitting the slow cooling rate of energetic carriers ${ }^{10}$. Such an enhanced phonon bottleneck effect facilitates the realization of the hot-carrier solar cell concept with an extremely high power conversion efficiency ${ }^{60}$.

In summary, we have precisely determined jumping rotational dynamics of molecules and phonons in hybrid perovskite $\mathrm{CH}_{3} \mathrm{NH}_{3} \mathrm{PbI}_{3}$ by conducting high-resolution QENS and INS measurements, which are all consistent with the finite-temperature MD simulations. The characteristic timescales of various dynamics are completely revealed. The dipole order of organic cations plays a decisive role in determining the physical properties such as carrier mobility and thermal conduction, distinguishing from inorganic perovskites. Such observations are probably general for all hybrid compounds with polar molecules. These fundamental insights are beneficial to further study of perovskite solar cells.

\section{Methods}

Single crystal growth and mounting. The crystal was grown by using the solution method described in ref. 6 . The single crystal used in neutron measurements is $2.32 \mathrm{~g}$ in weight and well-faceted. The as-grown single crystal was sealed into a plastic package under vacuum for storage and transportation. The crystal was mounted onto an aluminium holder in a glove box under helium atmosphere. They were sealed into an aluminium can using indium wire in the glove box. The whole process was completed without exposure to air to minimize the contamination by humidity and oxygen. The details of the sample are given in Supplementary Information, including Supplementary Figs 1, 11 and 12.

QENS and INS measurements. The high-resolution QENS and INS measurements were performed at the inverse-geometry time-of-flight chopper spectrometer BL02 DNA with $E_{\mathrm{i}}=2.084 \mathrm{meV}$ and at the direct-geometry time-of-flight chopper spectrometer BL14 AMATERAS with $E_{\mathrm{i}}$ of $54,16,7$ and $4 \mathrm{meV}$ of J-PARC in Japan $^{61,62}$. The instrumental resolutions are summarized in Supplementary Table 1 . The crystal was aligned at room temperature at DNA. It took $12 \mathrm{~h}$ to cool down to $140 \mathrm{~K}$ from room temperature at which it crystallizes in the tetragonal structure. At base temperature of $10 \mathrm{~K}$, the width of the elastic peak is $\sim 0.0036 \mathrm{meV}$, which is equal to the instrumental resolution of DNA. The crystal is twined in the orthorhombic phase, as shown in Fig. 4e and Supplementary Fig. 11. The four-dimension $S(\mathrm{q}, E)$ data were reduced and visualized by using Utsusemi suite $^{63}$, along $[h h 0],[00 l]$ and $[k \bar{k} 0]$ based on a tetragonal unit cell $(a=8.80625 \AA$, $c=12.712 \AA)$. The component along $[k \bar{k} 0]$ is limited by setting $-0.1<k<0.1$. The one-dimension spectra were fitted in PAN of DAVE ${ }^{64}$.

MD simulations. MD simulations were performed using the massively parallelized LAMMPS package ${ }^{65}$ and the recently developed classical MYP force field ${ }^{34}$, which reasonably reproduces the physical properties of $\mathrm{CH}_{3} \mathrm{NH}_{3} \mathrm{PbI}_{3}$ (refs $38,66,67$ ). In such a force field, non-bonded pairwise interactions are described by the sum of Buckingham, Lennard-Jones and long-range electrostatic potentials. Intra- and inter-molecular interactions of organic cations $\mathrm{CH}_{3} \mathrm{NH}_{3}^{+}$are described by the AMBER force field ${ }^{68}$. The cut-off distances for all the interactions are chosen to be $10 \AA$. A time step of $0.5 \mathrm{fs}$ was used in all simulations. After energy minimization, simulation models were first equilibrated for 250 ps under the NPT ensemble with a constant pressure of 1 bar and at various temperatures (100, 140, 180 and $200 \mathrm{~K})$. Then, they were simulated for another 50 ps under the NPT ensemble to calculate the DOS by taking the discrete Fourier transform of the mass-weighted VACFs of atoms, specifically given as ${ }^{69}$ :

$$
D(f)=\int_{0}^{\tau}\left(\sum_{i=1}^{N} m_{i} \frac{\left\langle\mathbf{v}_{i}(t) \cdot \mathbf{v}_{i}(0)\right\rangle}{\left\langle\mathbf{v}_{i}(0) \cdot \mathbf{v}_{i}(0)\right\rangle}\right) \mathrm{e}^{-i 2 \pi f t} \mathrm{~d} t
$$

where $f$ is the phonon vibration frequency; $\mathbf{v}_{i}(t)$ and $\mathbf{v}_{i}(0)$ are the atomistic velocities of atom $i$ at time $t$ and 0 , which are collected every $10 \mathrm{fs} ; m_{i}$ is the mass of atom $i$; $N$ is the total number of atoms belonging to certain species $\left(\mathrm{CH}_{3} \mathrm{NH}_{3}^{+}\right.$or $\left.\mathrm{PbI}_{3}^{-}\right) ; \tau=50 \mathrm{ps}$ is the auto-correlation time period used for data sampling; the angular brackets denote the time-averaged velocity auto-correlation functions. The Savitzky-Golay filter was employed to eliminate the thermal noise (Supplementary Fig. 13).

Data availability. The data that support the findings of this study are available from the corresponding authors upon reasonable request.

\section{References}

1. Brenner, T. M., Egger, D. A., Kronik, L., Hodes, G. \& Cahen, D. Hybrid organic-inorganic perovskites: low-cost semiconductors with intriguing charge-transport properties. Nat. Rev. Mater. 1, 15007 (2016).

2. Dong, Q. F. et al. Electron-hole diffusion lengths $>175 \mu \mathrm{m}$ in solution-grown $\mathrm{CH}_{3} \mathrm{NH}_{3} \mathrm{PbI}_{3}$ single crystals. Science 347, 967-970 (2015).

3. Shi, D. et al. Low trap-state density and long carrier diffusion in organolead trihalide perovskite single crystals. Science 347, 519-522 (2015)

4. Kojima, A., Teshima, K., Shirai, Y. \& Miyasaka, T. Organometal halide perovskites as visible-light sensitizers for photovoltaic cells. J. Am. Chem. Soc. 131, 6050-6051 (2009).

5. Yang, W. S. et al. High-performance photovoltaic perovskite layers fabricated through intramolecular exchange. Science 348, 1234-1237 (2015).

6. Liu, Y. et al. Two-inch-sized perovskite $\mathrm{CH}_{3} \mathrm{NH}_{3} \mathrm{PbX}_{3}(\mathrm{X}=\mathrm{Cl}, \mathrm{Br}, \mathrm{I})$ crystals: growth and characterization. Adv. Mater. 27, 5176-5183 (2015).

7. Liu, Y. et al. Thinness- and shape-controlled growth for ultrathin singlecrystalline perovskite wafers for mass production of superior photoelectronic devices. Adv. Mater. 28, 9204-9209 (2016).

8. Price, M. et al. Hot carrier cooling and photo-induced refractive index changes in organic-inorganic lead halide perovskites. Nat. Commun. 6, 8420 (2015)

9. Yang, Y. et al. Observation of a hot-phonon bottleneck in lead-iodide perovskites. Nat. Photon. 10, 53-59 (2016).

10. Yang, J. et al. Acoustic-optical phonon up-conversion and hot-phonon bottleneck in lead-halide perovskites. Nat. Commun. 8, 14120 (2017).

11. Sze, S. M. \& Ng, K. K. Physics of Semiconductor Devices (Wiley, 2007).

12. Blakemore, J. Semiconducting and other major properties of gallium arsenide. J. Appl. Phys. 53, R123 (1982).

13. Karakus, M. et al. Phonon-electron scattering limits free charge mobility in methylammonium lead iodide perovskites. J. Phys. Chem. Lett. 6, 4991-4996 (2015).

14. Wright, A. D. et al. Electron-phonon coupling in hybrid lead halide perovskites. Nat. Commun. 7, 11755 (2016).

15. Filippetti, A., Mattoni, A., Caddeo, C., Saba, M. I. \& Delugas, P. Low electron-polar optical phonon scattering as a fundamental aspect of carrier mobility in methylammonium lead halide $\mathrm{CH}_{3} \mathrm{NH}_{3} \mathrm{PbI}_{3}$ perovskites. Phys. Chem. Chem. Phys. 18, 15352-15362 (2016).

16. Pisoni, A. et al. Ultra-low thermal conductivity in organic-inorganic hybrid perovskite $\mathrm{CH}_{3} \mathrm{NH}_{3} \mathrm{PbI}_{3}$. J. Phys. Chem. Lett. 5, 2488-2492 (2014).

17. Wada, H. \& Kamijoh, T. Theraml conducitivity of amorphous silicon. Jpn J. Appl. Phys. 35, L648 (1996).

18. Lee, J.-H., Bristowe, N. C., Bristowe, P. D. \& Cheetham, A. K. Role of hydrogenbonding and its interplay with octahedral tilting in $\mathrm{CH}_{3} \mathrm{NH}_{3} \mathrm{PbI}_{3}$. Chem. Commun. 51, 6434-6437 (2015).

19. Ren, Y. et al. Orientation of organic cations in hybrid inorganic-organic perovskite $\mathrm{CH}_{3} \mathrm{NH}_{3} \mathrm{PbI}_{3}$ from subatomic resolution single crystal neutron diffraction structural studies. Cryst. Growth Design 16, 2945 (2016).

20. Motta, C. et al. Revealing the role of organic cations in hybrid halide perovskite $\mathrm{CH}_{3} \mathrm{NH}_{3} \mathrm{PbI}_{3}$. Nat. Commun. 6, 7026 (2015).

21. Drużbicki, K. et al. Unexpected cation dynamics in the low-temperature phase of methylammonium lead iodide: the need for improved models. J. Phys. Chem. Lett. 7, 4701-4709 (2016).

22. Brivio, F. et al. Lattice dynamics and vibrational spectra of the orthorhombic, tetragonal, and cubic phases of methylammonium lead iodide. Phys. Rev. B 92, 144308 (2015).

23. Pérez-Osorio, M. A. et al. Vibrational properties of the organic-inorganic halide perovskite $\mathrm{CH}_{3} \mathrm{NH}_{3} \mathrm{PbI}_{3}$ from theory and experiment: factor group analysis, first-principles calculations, and low-temperature infrared spectra. J. Phys. Chem. C 119, 25703-25718 (2015).

24. Mattoni, A., Filippetti, A., Saba, M. I., Caddeo, C. \& Delugas, P. Temperature evolution of methylammonium trihalide vibrations at the atomic scale. J. Phys. Chem. Lett. 7, 529-535 (2016).

25. Yue, S.-H. et al. Insight into the collective vibrational modes driving ultralow thermal conductivity of perovskite solar cells. Phys. Rev. B 94, 115427 (2016).

26. Leguy, A. M. A. et al. Dynamic disorder, phonon lifetimes, and the assigenment of the modes to the vibrational spectra of methylammonium lead halides perovskites. Phys. Chem. Chem. Phys. 18, 27051 (2016).

27. Bée, M. Quasielastic Neutron Scattering Principles And Applications In Solid State Chemistry, Biology And Materials Science (IOP Publishing Ltd, 1988). 
28. Weller, M. T. et al. Complete structure and cation orientation in the perovskite photovoltaic methylammonium lead iodide between 100 and $352 \mathrm{~K}$. Chem. Commun. 51, 4180-4183 (2015).

29. Whitfield, P. S. et al. Structures, phase transitions and tricritical behavior of the hybrid perovskite methylammonium lead iodide. Sci. Rep. 6, 35685 (2016).

30. Leguy, A. M. A. et al. The dynamics of methylammonium ions in hybrid organic-inorganic perovskite solar cells. Nat. Commun. 6, 7124 (2015).

31. Chen, T. et al. Rotational dynamics of organic cations in the $\mathrm{CH}_{3} \mathrm{NH}_{3} \mathrm{PbI}_{3}$ perovskite. Phys. Chem. Chem. Phys. 17, 31278 (2015).

32. Christians, J. A., Miranda Herrera, P. A. \& Kamat, P. V. Transformation of the excited state and photovoltaic efficiency of $\mathrm{CH}_{3} \mathrm{NH}_{3} \mathrm{PbI}_{3}$ perovskite upon controlled exposure to humidified air. J. Am. Chem. Soc. 137, 1530-1538 (2015).

33. Aristidou, N. et al. The role of oxygen in the degradation of methylammonium lead trihalide perovskite photoactive layers. Angew. Chem. Int. Ed. 54, 8208-8212 (2015).

34. Mattoni, A., Filippetti, A., Saba, M. I. \& Delugas, P. Methylammonium rotational dynamics in lead halide perovskite by classical molecular dynamics: the role of temperature. J. Phys. Chem. C 119, 17421-17428 (2015).

35. Whalley, L. D., Skelton, J. M., Frost, J. M. \& Walsh, A. Phonon anharmonicity, lifetimes, and thermal transport in $\mathrm{CH}_{3} \mathrm{NH}_{3} \mathrm{PbI}_{3}$ from many-body perturbation theory. Phys. Rev. B 94, 220301(R) (2016).

36. Comin, R. et al. Lattice dynamics and the nature of structural transitions in organolead halide perovskites. Phys. Rev. B 94, 094301 (2016).

37. Guo, Z., Yoon, S. J., Manser, J. S., Kamat, P. V. \& Luo, T. Structural phase- and degradation-dependent thermal conductivity of $\mathrm{CH}_{3} \mathrm{NH}_{3} \mathrm{PbI}_{3}$ perovskite thin films. J. Phys. Chem. C. 120, 6394-6401 (2016).

38. Wang, M. \& Lin, S. Anisotropic and ultralow phonon thermal transport in organic-inorganic hybrid perovskites: atomistic insights into solar cell thermal management and thermoelectric energy conversion efficiency. Adv. Funct. Mater. 26, 5297-5306 (2016).

39. Akhmadullin, I. S. h. \& Saburova, R. V. Phonon scattering by paraelectrical centers in crystals I. general. Phys. Stat. Solidi B 112, 367-378 (1982).

40. Akhmadullin, I. S. h. \& Saburova, R. V. Phonon scattering by paraelectrical centers in crystals II. lowest order approximation. Phys. Stat. Solidi B 113, 137-145 (1982)

41. Mante, P.-A. et al. Electron-acoustic phonon coupling in single crystal $\mathrm{CH}_{3} \mathrm{NH}_{3} \mathrm{PbI}_{3}$ perovskites revealed by coherent acoustic phonons. Nat. Commun. 8, 14398 (2017)

42. Lu, Y.-B. et al. Piezoelectric scattering limited mobility of hybrid organicinorganic perovskites $\mathrm{CH}_{3} \mathrm{NH}_{3} \mathrm{PbI}_{3}$. Sci. Rep. 7, 41860 (2017).

43. Gélvez-Rueda, M. C. et al. Effect of cation rotation on charge dynamics in hybrid lead halide perovskites. J. Phys. Chem. C 120, 16577-16585 (2016).

44. Onoda-Yamamuro, N., Matsuo, T. \& Suga, H. Dielectric study of $\mathrm{CH}_{3} \mathrm{NH}_{3} \mathrm{PbX}_{3}$ $(\mathrm{X}=\mathrm{Cl}, \mathrm{Br}, \mathrm{I})$. J. Phys. Chem. Solids 53, 935-939 (1992).

45. Wemple, S. H., DiDomenico, Jr. M. \& Jayaraman, A. Electron scattering in perovskite-oxide ferroelectric semiconductors. Phys. Rev. 180, 547 (1969).

46. Katayama, S. \& Mills, D. L. Theory of anomalous resistivity associated with structural phase transitions in IV-VI compounds. Phys. Rev. B 22, 336 (1980).

47. Binder, K., Meissner, G. \& Mais, H. Equation of state, Debye-Waller factor, and electrical resistivity of ferroelectrics near their critical point. Phys. Rev. B 13, 4890 (1976).

48. Maiorano, A., Martín-Mayor, V., Ruiz-Lorenzo, J. J. \& Tarancón, A. Weak firstorder transition in the three-dimensional site-diluted Ising antiferromagnet in a magnetic field. Phys. Rev. B 76, 064435 (2007).

49. Lahnsteiner, J. et al. Room-temperature dynamic correlation between methylammonium molecules in lead-iodine based perovskites: an ab initio molecular dynamics perspective. Phys. Rev. B 94, 214114 (2016).

50. Frost, J. M. et al. Atomistic origins of high-performance in hybrid halide perovskite solar cells. Nano Lett. 14, 2584-2590 (2014).

51. Kutes, Y. et al. Direct observation of ferroelectric domains in solutionprocessed $\mathrm{CH}_{3} \mathrm{NH}_{3} \mathrm{PbI}_{3}$ perovskite thin films. J. Phys. Chem. Lett. 5, 3335-3339 (2014).

52. Zhu, X.-Y. \& Podzorov, V. Charge carriers in hybrid organic-inorganic lead halide perovskites might be protected as large polarons. J. Phys. Chem. Lett. 6, 4758-4761 (2015).

53. Tritt, T. M. Thermal Conductivity: Theory, Properties, And Applications (Klumer Academic/Plenum Publishers, 2004).

54. Caddeo, C. et al. Tuning the thermal conductivity of methylammonium lead halide by the molecular substructure. Phys. Chem. Chem. Phys. 18, 24318-24324 (2016).

55. Hata, T., Giorgi, G. \& Yamashita, K. The Effects of the organic-inorganic interactions on the thermal transport properties of $\mathrm{CH}_{3} \mathrm{NH}_{3} \mathrm{PbI}_{3}$. Nano Lett. 16, 2749-2753 (2016)

56. Slack, G. A. in CRC Handbook of Thermoelectrics (ed. Rowe, D. M.) 407-440 (CRC, 1995)

57. Cahill, D. G. \& Pohl, R. O. Lattice vibrations and heat transport in crystals and glasses. Ann. Rev. Phys. Chem. 39, 93-121 (1988).
58. Filippetti, A., Caddeo, C., Delugas, P. \& Mattoni, A. Appealing perspectives of hybrid lead-iodide perovskites as thermoelectric materials. J. Phys. Chem. C 120, 28472-28479 (2016)

59. Mettan, X. et al. Tuning of the thermoelectric figure of merit of $\mathrm{CH}_{3} \mathrm{NH}_{3} \mathrm{MI}_{3}$ $(\mathrm{M}=\mathrm{Pb}, \mathrm{Sn})$ photovoltaic perovskites. J. Phys. Chem. C 119, 11506-11510 (2015).

60. Zhu, H. et al. Screening in crystalline liquids protects energetic carriers in hybrid perovskites. Science 353, 1409-1413 (2016).

61. Shibata, K. et al. The performance of TOF near backscattering spectrometer DNA in MLF, J-PARC. JPS Conf. Proc. 8, 036022 (2015).

62. Nakajima, K. et al. AMATERAS: a cold-neutron disk chopper spectrometer. J. Phys. Soc. Jpn 80, SB028 (2011).

63. Inamura, Y. et al. Development status of software "Utsusemi" for chopper spectrometers at MLF, J-PARC. J. Phys. Soc. Jpn 82, SA031 (2013).

64. Azuah, R. T. et al. DAVE: a comprehensive software suite for the reduction, visualization, and analysis of low energy neutron spectroscopic data. J. Res. Natl Inst. Stand. Technol. 114, 341-358 (2009).

65. Plimpton, S. Fast parallel algorithms for short-range molecular dynamics. J. Comp. Phys. 117, 1-19 (1995).

66. Yu, J., Wang, M. \& Lin, S. Probing the soft and nanoductile mechanical nature of single and polycrystalline organic-inorganic hybrid perovskites for flexible functional devices. ACS Nano 10, 11044-11057 (2016).

67. Mattoni, A., Filippetti, A. \& Caddeo, C. Modeling hybrid perovskites by molecular dynamics. J. Phys. Condens. Matter 29, 043001 (2017).

68. Ponder, J. W. \& Case, D. A. Force fields for protein simulations. Adv. Protein Chem. 66, 27-85 (2003).

69. Lin, S. \& Buehler, M. J. The effect of non-covalent functionalization on the thermal conductance of graphene/organic interfaces. Nanotechnology 24, 165702 (2013).

\section{Acknowledgements}

We acknowledge the award of beam time from J-PARC via proposals, No. 2015A0075 and 2012P0906. Y.L. and S.(F.)L. were supported by the National Natural Science Foundation of China (61674098), the National Key Research project MOST (2016YFA0202400 and 2017YFA0204800), the 111 Project (B14041), National University Research Fund (GK261001009 and GK201603107) and the Chinese National 1000-talent-plan program (1110010341). M.W. and S.L. were supported by the startup funding from the Energy and Materials Initiative of the Florida State University. We thank Dr M. Kofu, Dr R. Kajimoto, Dr M. Nakamura, Dr T. Ohhara and Dr R. Kiyanagi for fruitful discussion.

\section{Author contributions}

B.L. proposed the project. Y.L. and S.(F.)L. synthesized the sample. B.L., Y.K., M.M., K.S., S.O-K., T.Y. and K.N. carried out the QENS and INS measurements. M.W. and S.L. performed MD simulations. B.L. analysed all experimental data. B.L. and S.(F.)L. wrote the manuscript with discussion and input from all the authors.

\section{Additional information}

Supplementary Information accompanies this paper at http://www.nature.com/ naturecommunications

Competing interests: The authors declare no competing financial interests.

Reprints and permission information is available online at http://npg.nature.com/ reprintsandpermissions/

How to cite this article: Li, B. et al. Polar rotor scattering as atomic-level origin of low mobility and thermal conductivity of perovskite $\mathrm{CH}_{3} \mathrm{NH}_{3} \mathrm{PbI}_{3}$. Nat. Commun. 8, 16086 doi: 10.1038/ncomms16086 (2017).

Publisher's note: Springer Nature remains neutral with regard to jurisdictional claims in published maps and institutional affiliations.

Open Access This article is licensed under a Creative Commons Attribution 4.0 International License, which permits use, sharing, adaptation, distribution and reproduction in any medium or format, as long as you give appropriate credit to the original author(s) and the source, provide a link to the Creative Commons license, and indicate if changes were made. The images or other third party material in this article are included in the article's Creative Commons license, unless indicated otherwise in a credit line to the material. If material is not included in the article's Creative Commons license and your intended use is not permitted by statutory regulation or exceeds the permitted use, you will need to obtain permission directly from the copyright holder. To view a copy of this license, visit http://creativecommons.org/ licenses/by/4.0/

(C) The Author(s) 2017 